\title{
Influences on pathologic complete response in breast cancer patients after neoadjuvant chemotherapy
}

\author{
Carolin Müller ${ }^{1} \mathbb{D}$. Gilda Schmidt ${ }^{1}$. Ingolf Juhasz-Böss ${ }^{2} \cdot$ Lisa Jung $^{2} \cdot$ Sarah Huwer $^{2} \cdot$ Erich-Franz Solomayer $^{1}$. \\ Stephanie Juhasz-Böss ${ }^{1}$
}

Received: 25 November 2020 / Accepted: 23 February 2021 / Published online: 10 March 2021

(c) The Author(s) 2021

\begin{abstract}
Purpose Pathologic complete response is associated with longer disease-free survival and better overall survival after neoadjuvant chemotherapy in breast cancer patients. We, therefore, evaluated factors influencing pathologic complete response. Methods Patients receiving neoadjuvant chemotherapy from 2015 to 2018 at the Saarland University Hospital were included. Patients' age, tumor stage, tumor biology, genetic mutation, recurrent cancer, discontinuation of chemotherapy, and participation in clinical trials were extracted from electronic medical records. Binary logistic regression was performed to evaluate the influence of these factors on pathologic complete response.

Results Data of 183 patients were included. The median patient's age was 54 years (22-78). The median interval between diagnosis and onset of chemotherapy was 28 days (14-91); between end of chemotherapy and surgery 28 days (9-57). Sixtytwo patients (34\%) participated in clinical trials for chemotherapy. A total of 86 patients (47\%) achieved pathologic complete response. Patient's age, genetic mutation, recurrent cancers, or discontinuation of chemotherapy (due to side effects) and time intervals (between diagnosis and onset of chemotherapy, as well as between end of chemotherapy and surgery) did not influence pathologic complete response. Patients with high Ki67, high grading, Her2 positive tumors, as well as patients participating in clinical trials for chemotherapy had a higher chance of having pathologic complete response. Patients with Luminal B tumors had a lower chance for pathologic complete response.

Conclusion Particularly patients with high risk cancer and patients, participating in clinical trials benefit most from chemotherapy. Therefore, breast cancer patients can be encouraged to participate in clinical trials for chemotherapy.
\end{abstract}

Keywords Breast cancer $\cdot$ Neo-adjuvant therapy $\cdot$ Pathologic complete response $\cdot$ Clinical trials

\section{Introduction}

Neoadjuvant chemotherapy (NACT) has changed its role for only inoperable and locally advanced breast cancer to a treatment used in early stages of breast cancer [1]. Particularly patients at high risk, like patients with Her2 positive and triple-negative breast cancer benefit from receiving NACT $[2,3]$. Pathological complete response (pCR) is associated

Carolin Müller

carolin.mueller@uks.eu

1 Department of Gynecology, Obstetrics and Reproductive Medicine, Saarland University Medical Center, Kirrbergerstraße 100, 66424 Homburg/Saar, Germany

2 Department of Gynecology, Obstetrics and Reproductive Medicine, University Medical Center Freiburg, Freiburg, Germany with a better outcome, meaning that patients having $\mathrm{pCR}$ have longer disease-free survival and better overall survival [5].

The definition of $\mathrm{pCR}$ varies in individual studies (ypT0/ ypTis/ypN0). However, it could be shown that no residual invasive cancer in the breast and axilla is associated with better outcomes compared with no residual cancer in the breast alone [4]. Von Minckwitz et al. even detected a longer disease-free survival in patients with no invasive and no in situ carcinoma in the breast and axilla [5]. Also, tumor subtypes influence the achievement of pCR and thus a better outcome. For example, LeVasseur et al. detected that patients with triple negative tumors had longer relapse-freesurvival and breast cancer-specific survival when achieving pCR after NACT [6].

As pCR plays an important role in the outcome of breast cancer, several studies already evaluated possible influencing 
factors, like age of the patients [7, 8], tumor biology [9] or genetic mutation [10]. The majority of the cited studies analyzed data that was previously collected as part of NACT trials. But patients who are included in clinical studies are mostly a selected collective due to in- and exclusion criteria with fewer previous illnesses, or of a certain subtype or tumor size. For this reason, we wanted to analyze possible influencing factors on pCR in "real-world-data".

Although, some factors such as age, tumor biology or genetic mutation cannot be influenced directly, it is still important to identify possible risk factors to maybe establish further follow-up therapies or intensified aftercare. We, therefore, determined various clinical factors from electronic patient records and evaluated their influence on the probability of pCR. Parts of the registry were used for two other analyzes before $[11,12]$. We could show that especially inpatient and outpatient presentations of the patients delayed therapy onset of NACT and surgery [11, 12].

\section{Patients and methods}

\section{Data collection}

All patients receiving NACT due to newly diagnosed breast cancer between 2015 to 2018 at the Department of Gynecology, Saarland University Medical Center, were included in the study. The primary endpoint was pCR after NACT. pCR was defined as no residual tumor in the breast and axilla (ypT0, N0). It includes no residual Tis (Carcinoma in situ). Date of diagnosis was defined as the date of core biopsy. Patients' age, tumor stage, eventual multi-centricity, and tumor biology were recorded. It was distinguished whether the patients received NACT as standard therapy or in a clinical trial. Mainly GAIN II [13], Gepar Octo [14], and Gepar X [15] were addressed from 2015 to 2018 in the Saarland University Medical Center as clinical trials. The time interval between diagnosis and onset of chemotherapy, as well as between end of chemotherapy and surgery, was recorded. The type of operation and duration of hospitalization were documented. Patients who had an indication for BRCA testing according to the guidelines of the German AGO Mamma and the German Consortium for Hereditary Breast and Ovarian Cancer (GC-HBOC) were tested for a BRCA mutation. Their recommendations are based on an analysis of 21,401 families [16]. It was registered whether patients had a genetic mutation (BRCA) or recurrent cancer. Recurrent cancer was defined as local recurrence of breast cancer. Patients with local recurrence were included in the study regardless of their previous therapy. If patients discontinued chemotherapy due to therapy complications or disease progress, it was recorded as well.

\section{Data management and statistics}

Patients' data were reviewed in the hospital's digital documentation system (SAP, Walldorf, Germany). Data were collected using Microsoft Excel $2010^{\circledR}$ (Microsoft, Redmond, USA). Further statistics were performed with SPSS 24.0 (IBM, Armonk, USA). Quantitative parameters (e.g., patients age, days) are given as median and range. Qualitative parameters (e.g., tumor stage) are presented as frequencies. Binary logistic regression was performed to determine the influence of multiple factors on pCR. Possible influencing factors were age, study participation, tumor biology, genetic mutation (BRCA), recurrent cancer, discontinuation of chemotherapy (due to side effects), days between diagnosis and begin of chemotherapy as well as days between end of chemotherapy and operation. All procedures performed in the study involving human participants were in accordance with the ethical standards of the institutional and national research committee and with the 1964 Helsinki Declaration and its later amendments or comparable ethical standards. Informed consent was obtained from every individual participant included in the study.

\section{Results}

Between 2015 and 2018, a total of 205 patients received NACT due to newly diagnosed breast cancer at the Saarland University Medical Center. Twenty-two patients had to be excluded because of insufficient data so that the data of 183 patients were analyzed. The patients median age was 54 years (22-78). The median time between diagnosis and begin of NACT was 28 days (14-91). Between the end of NACT and operation, median time was 28 days (9-57).

Tumor stage and biology, as well as histological subtype and grading are illustrated in Table 1. Twenty-eight patients $(15 \%)$ had multicentricity. BRCA mutation (9 patients, $5 \%$ ) and recurrent cancers (7 patients, $4 \%$ ) were rare. Sixty-two patients (34\%) received study therapies: GAIN II (12\%), Gepar Octo (10\%), GeparX (9\%), others (5\%). Patients participating in clinical studies had a higher percentage of triple negative tumors (42\%) compared to patients without study treatment $(26 \%)$. Her2 positive tumors are less frequent in the study group (34\%), compared to standard therapy regimen (39\%). Furthermore, Grading and Ki67 values are higher in patients participating in clinical trials (Grading G3 67\% vs. 53\%, Ki67 $50 \%$ vs. $40 \%$ ). All patients received an Anthracycline and Taxane-based chemotherapy. Fifty-four patients of the Her2 positive group (79\%) received chemotherapy 
Table 1 Tumor characteristics

\begin{tabular}{|c|c|c|}
\hline TNM-stage & Absolute frequencies $(n)$ & $\begin{array}{l}\text { Cumulative } \\
\text { frequencies } \\
(\%)\end{array}$ \\
\hline \multicolumn{3}{|l|}{ Staging } \\
\hline \multicolumn{3}{|l|}{ ypT } \\
\hline 0 & 89 & 48.6 \\
\hline 1 & 56 & 30.6 \\
\hline 2 & 16 & 8.7 \\
\hline 3 & 7 & 3.8 \\
\hline 4 & 5 & 2.7 \\
\hline $\mathrm{X}$ & 10 & 5.6 \\
\hline \multicolumn{3}{|l|}{ ypN } \\
\hline 0 & 56 & 30.6 \\
\hline 1 & 26 & 14.2 \\
\hline 2 & 15 & 8.2 \\
\hline 3 & 3 & 1.6 \\
\hline \multicolumn{3}{|l|}{ Pre-therapeutic } \\
\hline pN0 (sentinal node) & 83 & 45.4 \\
\hline $\mathrm{pN}+$ & 100 & 54.6 \\
\hline Tumor biology & Absolute frequencies $(n)$ & $\begin{array}{l}\text { Cumulative } \\
\text { frequencies } \\
(\%)\end{array}$ \\
\hline \multicolumn{3}{|l|}{ Tumor biology } \\
\hline NST (invasive carcinoma of no special type) & 173 & 94.5 \\
\hline Others & 10 & 5.5 \\
\hline Histological subtype & Absolute frequencies $(n)$ & $\begin{array}{l}\text { Cumulative } \\
\text { frequencies } \\
(\%)\end{array}$ \\
\hline
\end{tabular}

\section{Histology}

Luminal A

HR+, Her2 neg, Ki67 $\leq 15 \%$

$8 \quad 4.4$

Luminal B

$\begin{array}{lll}\text { HR+, Her2 neg, Ki67> } 15 \% & 50 & 27.3\end{array}$

Her2 positive, HR positive $\quad 48 \quad 26.2$

Her2 positive, HR negative $\quad 20 \quad 11.0$

$\begin{array}{lll}\text { Triple negative } & 57 & 31.1\end{array}$

$\begin{array}{lll}\text { Grading } & \text { Absolute frequencies }(n) & \text { Cumulative }\end{array}$

\section{Grading}

G1

G2

$\begin{array}{rr}2 & 1.1 \\ 74 & 40.4 \\ 105 & 57.4 \\ 2 & 1.1\end{array}$

$\mathrm{X}$

$H R$ hormone receptor

in combination with Trastuzumab and Pertuzumab, $21 \%$ received chemotherapy in combination with Trastuzumab alone. All patients with triple-negative carcinoma additionally received carboplatin. Thirty-four patients (19\%) discontinued NACT. Twenty patients (11\%) skipped only the last or the last two chemotherapy doses, whereas 14 patients $(8 \%)$ received three or more than three doses less. The reasons for a premature discontinuation of 
chemotherapy were equally distributed in both groups; the major part (16\%) due to side effects (polyneuropathy $7 \%$, changes in laboratory values as increased liver values or cytopenia $6 \%$, others $3 \%$ ). Four patients (2\%) discontinued NACT because of tumor progression.

Most patients received breast-conserving therapy (63\%), followed by mastectomy (22\%) and oncoplastic surgery (15\%). Implants or expander was used for 24 patients (13\%). The median time of hospitalization during the operation was 4 days (1-22). Patients with breast-conserving therapy had a median time of hospitalization of 3 days (1-14), patients undergoing mastectomy 6 days (2-17) and patients with oncoplastic surgery 7 days (2-22).

A total of 86 patients (47\%) had pCR. The patients age, genetic mutation, recurrent cancer, or discontinuation of chemotherapy (due to side effects) did not influence pCR. Likewise, time between diagnosis and onset of NACT, or time between end of NACT and surgery, had no influence on pCR. Patients participating in clinical trials for NACT, higher tumor grade, high Ki67 and Her2 positive tumors had increased chances of having pCR. Patients with Luminal B tumors had a lower chance of achieving pCR. No pCR was detected in the Luminal A group. In patients with triple negative tumors, a trend could be observed. They seem to have more often $\mathrm{pCR}$, although not statistically significant. Influences on pCR are shown in Table 2.

Table 2 Influences on pCR by binary logistic regression

\begin{tabular}{llrl}
\hline Parameter & $\begin{array}{l}\text { Odds ratio } \\
\text { Exp }(B)\end{array}$ & $P$ & $\begin{array}{l}95 \% \text { confidence } \\
\text { interval }\end{array}$ \\
\hline Age & 1.022 & 0.096 & $0.996-1.048$ \\
Time interval & 0.990 & 0.450 & $0.965-1.016$ \\
Diagnosis-NACT & & 0.285 & $0.949-1.016$ \\
Time interval & 0.982 & & \\
NACT-operation & & $\mathbf{0 . 0 0 9}$ & $1.244-4.458$ \\
Study participation & 2.355 & 0.275 & $0.533-9.103$ \\
Genetic mutation & 2.203 & 0.294 & $0.077-2.171$ \\
Recurrent cancers & 0.410 & 0.115 & $0.852-4.371$ \\
Discontinuation of & 1.929 & & \\
$\quad$ NACT & 1.031 & $<\mathbf{0 . 0 0 1}$ & $1.016-1.047$ \\
Ki67 & 4.201 & $<\mathbf{0 . 0 0 1}$ & $2.206-7.999$ \\
Grading & 1.493 & 0.219 & $0.788-2.827$ \\
Triple negative & 2.053 & $\mathbf{0 . 0 4 5}$ & $1.015-4.150$ \\
Her2 positive & & & \\
HR positive & 4.500 & $\mathbf{0 . 0 0 6}$ & $1.526-13.273$ \\
Her2 positive & & & \\
HR negative & 0.297 & $\mathbf{0 . 0 0 1}$ & $0.144-0.616$ \\
Luminal B & & & \\
\hline
\end{tabular}

Bold values represents statistically significant

\section{Discussion}

This study presents several influences on pCR, with increased chances in patients participating in studies for NACT, having a high Ki67, high grading or Her2 positive tumors. Tumor subtypes like Luminal A and B had decreased chances of pCR.

Age had no influence on pCR in the current study. In contrast, other studies reported that young age is positively associated with pCR [7]. A cutoff value of 40 years was proposed under which the chances of pCR may be higher [17]. However, pCR can also be achieved in elderly patients, especially for Her2 positive patients [8]. So, age alone should not be an indication for NACT.

Neither time between diagnosis and onset of NACT, nor time between the end of chemotherapy and surgery influenced pCR. Consistently, exceeding a presumed cut-off of 4 weeks for onset of NACT or surgery did not influence pCR and disease-free or overall survival independent of histopathological subgroups [18]. However, it was suspected that patients without pCR could benefit from early surgery [18]. At least time intervals up to 8 weeks between NACT and surgery seem not to influence outcomes [19]. In the present study, the median time from diagnosis to NACT and NACT to surgery was about a month with only a minor part exceeding 8 weeks, which complies with German guidelines [20]. This limits the ability to assess the influence of longer time intervals on pCR. However, our results suggest that therapy intervals seem to have a negligible influence on $\mathrm{pCR}$.

More than a third of our patients participated in clinical trials. Study participants for NACT had more than twice as high a chance to achieve pCR. In addition to higher chances for $\mathrm{pCR}$ in patients participating in clinical trials, mastectomy rates were lower [21]. However, one must take into account that the patients who are included in clinical studies are mostly a selected collective. On the one hand, this means that there is a disproportionate part of those patients who have a higher probability to achieve pCR due to tumor biology (e.g. high grading and high Ki67). On the other hand, study patients have fewer previous illnesses due to in- and exclusion criteria which can otherwise lead to premature discontinuation of therapy, dose reduction or longer therapy breaks. In the present study, patients receiving NACT in clinical trials had higher rates of Ki67 and higher grading compared to patients receiving standard therapy. This might be the reason for higher pCR rates in the study participants. Furthermore, there was a higher percentage with triple negative tumors in the study group comparing to standard treatment. However, the rate of Her2 positive tumors (which are also associated with higher rates of $\mathrm{pCR}$ ) was lower in patients participating 
in clinical trials. Nevertheless, we should encourage our patients to participate in clinical trials. Besides contributing to therapy improvements, they most likely benefit from new therapies. Possible disadvantages of trial participation, such as delaying the onset of NACT or surgery, could be excluded [11, 12].

Only $5 \%$ of our patients had a proven genetic mutation (BRCA) for which a superior response to NACT was previously described [10]. Particularly when BRCA positive patients have triple-negative tumors, better outcomes are suspected [22]. We could not prove a significant influence of genetic mutation on pCR.

Likewise, there was no influence of local recurrent cancers on pCR. To our knowledge, this is the first study analyzing a possible influence of local recurrent cancers on the pCR rate. However, no distinction was made whether patients already received prior chemotherapy. In contrast to that, there are analyses describing the association of pCR after NACT on the recurrence of locoregional cancer. An analysis of 10,075 women showed that the local recurrence rate after chemotherapy was higher in patients with non-pCR (9.5\% in 67 months) [23].

Discontinuation of chemotherapy due to intolerable side effects did not influence pCR. Patients discontinuing NACT due to disease progress were not included. In general, guidelines recommend the completion of chemotherapy [20]. Our results imply that discontinuation of chemotherapy may not substantially lower chances for pCR. However, it should be considered that this might be due to the small sample size and that most patients only discontinued before the last or the last two chemotherapy doses. Nevertheless, a detrimental effect of early termination of chemotherapy cannot be excluded. Peripheral neurotoxicity was the most common reason why patients had to discontinue chemotherapy (7\%). Unfortunately, therapeutic options like pharmacological treatments are limited [24]. Thus, only dose reduction or discontinuation are current options [24]. Nevertheless, premature discontinuation of NACT seems to be uncritical, especially when a good clinical response was already proven. Further studies are needed to confirm our findings.

Ki67 and high grading were associated with high pCR rates $(P<0.001)$. As Ki67 increases by 1 , the probability of a pCR increases by 1.031 . This is consistent with findings that especially highly aggressive tumors have a good response to chemotherapy [5, 25]. Triple-negative tumors tend to have better pCR rates without reaching statistical significance. Other studies already showed that triple-negative tumors (as aggressive tumors) were associated with higher pCR rates after NACT [5, 7]. Her2 positive tumors showed higher chances for $\mathrm{pCR}$ in our study. This has also been demonstrated in previous studies [26, 27]. It could be shown that patients with Her2 positive, hormone receptor (HR) negative tumors showed the highest rates of pCR. With this tumor biology (Her2+, HR-) the chance to achieve pCR was 4.5 times higher than in other tumor subtypes. Von Minckwitz et al. and Harbeck et al. also observed that Her2 positive tumors respond particularly well to NACT when hormone receptor status is negative [5, 28]. In the present study, even tumors with Her2 positive, hormone receptor positive tumors had a twice as high chance to achieve $\mathrm{pCR}$ $(P=0.045)$. This effect could not be shown in all previous studies [5]. One reason for this might be the change in Her2 directed therapy, as the addition of pertuzumab to trasuzumab and NACT led to a more frequent pCR rate [29, 30]. In the present study, most patients (79\%) received Trastuzumab and Pertuzumab. Only a minor part (21\%) received Trastuzumab alone.

The subtype Luminal B was associated with a lower chance for pCR. Available data seem contradictory. pCR seems to appear more likely in young patients with hormone receptor-positive, Her2 negative breast cancer [7]. However, large analyzes of 13,939 women showed that the lowest rate of pCR was achieved for Luminal A, followed by Luminal $\mathrm{B}$ subtypes, whereas $\mathrm{pCR}$ rates of triple-negative and Her2 positive were comparatively higher [27]. Also, patients with progesterone negativity showed higher pCR rates [9]. Taken together, high Ki67, high grading and Her2 positive tumors are notably associated with higher pCR. No pCR was detected in the Luminal A group. This seems not to be surprisingly, as Luminal A cancers are not appropriate for NACT and normally show the lowest rates of pCR [27]. In this analysis, the four patients with Luminal A cancers had locally advanced tumors and received chemotherapy therefore neoadjuvant.

This study has limitations. It is single centric, and thus clinical pathways may differ to other centers. However, data were collected from a certified tertiary breast care center, ensuring that therapies are state of the art. The sample size is small, but many other studies are based on registers obtained from clinical trials for new therapy regimes [5, 7-9, 18, 21], which implies selection bias due to specific inclusion criteria. Our data more likely represents "real-world-data".

\section{Conclusion}

Particularly patients with aggressive tumors (high Ki67, high grading, Her2 positive tumors) had better response rates on NACT. These patients should receive chemotherapy in a neoadjuvant setting. Furthermore, patients participating in clinical trials had higher $\mathrm{pCR}$ rates after NACT. Besides contributing to therapy improvements, they most likely benefit from new therapies. We should thus encourage our patients to participate in clinical trials. 
Author contribution All authors contributed to the study conception and design. Material preparation and data collection were performed by Carolin Müller, Lisa Jung and Sarah Huwer. The data analysis was performed by Carolin Müller. The first draft of the manuscript was written by Carolin Müller and all authors commented on previous versions of the manuscript. All authors read and approved the final manuscript.

Funding Open Access funding enabled and organized by Projekt DEAL.

Availability of data and material The datasets generated during and/or analysed during the current study are available from the corresponding author on reasonable request.

\section{Declarations}

Conflict of interest All of the authors (Carolin Müller, Gilda Schmidt, Stephanie Juhasz-Böss, Lisa Jung, Sarah Huwer, Erich-Franz Solomayer, Ingolf Juhasz-Böss) declare that they have no conflict of interest.

Ethical approval This research study was conducted retrospectively from data obtained for clinical purposes. All procedures performed in the study involving human participants were in accordance with the ethical standards of the institutional and/or national research committee (Ethics committee of the Saarland Physicians' chamber) and with the 1964 Helsinki Declaration and its later amendments or comparable ethical standards. Informed consent was obtained from every individual participant included in the study. Trial registration number: 207/10 (Ethics committee of the Saarland Physicians' chamber). This article does not contain any studies with animals performed by any of the authors.

Open Access This article is licensed under a Creative Commons Attribution 4.0 International License, which permits use, sharing, adaptation, distribution and reproduction in any medium or format, as long as you give appropriate credit to the original author(s) and the source, provide a link to the Creative Commons licence, and indicate if changes were made. The images or other third party material in this article are included in the article's Creative Commons licence, unless indicated otherwise in a credit line to the material. If material is not included in the article's Creative Commons licence and your intended use is not permitted by statutory regulation or exceeds the permitted use, you will need to obtain permission directly from the copyright holder. To view a copy of this licence, visit http://creativecommons.org/licenses/by/4.0/.

\section{References}

1. Teshome MD, Hunt KK (2014) Neoadjuvant therapy in the treatment of breast cancer. Surg Oncol Clin N AM 23:505-523. https ://doi.org/10.1016/j.soc.2014.03.006

2. Bartsch R, Bergen E, Galid A (2018) Current concepts and future directions in neoadjuvant chemotherapy of breast cancer. Memo Mag Eur Med Oncol 11:199-203. https://doi.org/10.1007/s1225 4-018-0421-1

3. Cortazar P, Zhang L, Untch M et al (2014) Pathological complete response and long-term clinical benefit in breast cancer: the CTNeoBC pooled analysis. Lancet 384:164-172. https://doi. org/10.1016/S0140-6736(13)62422-8
4. Cortazar P, Geyer CE (2015) Pathological complete response in neoadjuvant treatment of breast cancer. Ann Surg Oncol 22:14411446. https://doi.org/10.1245/s10434-015-4404-8

5. Von Minckwitz G, Untch M, Blohmer JU et al (2012) Definition and impact of pathologic complete response on prognosis after neoadjuvant chemotherapy in various intrinsic breast cancer subtypes. J Clin Oncol 30:1796-1804. https://doi.org/10.1200/ JCO.2011.38.8595

6. LeVasseur N, Sun J, Gondara L et al (2020) Impact of pathologic complete response on survival after neoadjuvant chemotherapy in early-stage breast cancer: a population-based analysis. J Cancer Res Clin Oncol 146:529-536. https://doi.org/10.1007/s0043 2-019-03083-y

7. Loibl S, Jackisch C, Lederer B et al (2015) Outcome after neoadjuvant chemotherapy in young breast cancer patients: a pooled analysis of individual patient data from eight prospectively randomized controlled trials. Breast Cancer Res Treat 152:377-387. https://doi.org/10.1007/s10549-015-3479-z

8. Von WG, Loibl S, Furlanetto J et al (2018) Outcome after neoadjuvant chemotherapy in elderly breast cancer patients-a pooled analysis of individual patient data from eight prospectively randomized controlled trials. Oncotarget 9:15168-15179

9. van Mackelenbergh MT, Denkert C, Nekljudova V et al (2018) Outcome after neoadjuvant chemotherapy in estrogen receptorpositive and progesterone receptor-negative breast cancer patients: a pooled analysis of individual patient data from ten prospectively randomized controlled neoadjuvant trials. Breast Cancer Res Treat 167:59-71. https://doi.org/10.1007/s10549-017-4480-5

10. Hahnen E, Lederer B, Hauke J et al (2017) Germline mutation status, pathological complete response, and disease-free survival in triple-negative breast cancer: secondary analysis of the GeparSixto randomized clinical trial. JAMA Oncol 3:1378-1385. https ://doi.org/10.1001/jamaoncol.2017.1007

11. Müller C, Juhasz-Böss S, Schmidt G et al (2019) Factors influencing the onset of neoadjuvant therapy in breast cancer patients. Breast Care. https://doi.org/10.1159/000502223

12. Müller C, Juhasz-Böss I, Schmidt G et al (2020) Factors influencing the time to surgery after neoadjuvant chemotherapy in breast cancer patients. Arch Gynecol Obstet. https://doi.org/10.1007/ s00404-020-05494-6

13. GAIN II. [cited 2020 Mar 25]. Available from clinicaltrials.gov/ ct2/show/NCT01690702

14. GeparOcto. [cited 2020 Mar 25]. Availabe from clinicaltrials.gov/ ct $2 /$ show/NCT02125344

15. GeparX. [cited 2020 Mar 25]. Available from clinicaltrials.gov/ ct $2 /$ show/NCT02682693? cond $=$ geparX\&draw $=2 \&$ rank $=1$

16. Kast K, Rhiem K, Wappenschmidt B et al (2016) Prevalence of BRCA1/2 germline mutations in 21401 families with breast and ovarian cancer. J Med Genet 53:465-471. https://doi.org/10.1136/ jmedgenet-2015-103672

17. Huober J, Von Minckwitz G, Denkert C et al (2010) Effect of neoadjuvant anthracycline-taxane-based chemotherapy in different biological breast cancer phenotypes: overall results from the GeparTrio study. Breast Cancer Res Treat 124:133-140. https:// doi.org/10.1007/s10549-010-1103-9

18. Loibl S, Werutsky G, Nekljudova V et al (2017) Impact in delay of start of chemotherapy and surgery on pCR and survival in breast cancer: a pooled analysis of individual patient data from six prospectively randomized neoadjuvant trials. J Clin Oncol 35:571-571

19. Sanford RA, Lei $X$, Barcenas $\mathrm{CH}$ et al (2016) Impact of time from completion of neoadjuvant chemotherapy to surgery on survival outcomes in breast cancer patients. Ann Surg Oncol 23:15151521. https://doi.org/10.1245/s10434-015-5020-3 
20. AGO Breast Committee. Guidelines of neoadjuvant (primary) systemic therapy. recommendations 2020. [cited 25 Mar 2020]. Available from www.ago-online.de

21. Brennan M, Gass P, Häberle L et al (2018) The effect of participation in neoadjuvant clinical trials on outcomes in patients with early breast cancer outcomes. Breast Cancer Res Treat. https://doi. org/10.1007/s10549-018-4829-4

22. Spugnesi L, Gabriele M, Scarpitta R et al (2016) Germline mutations in DNA repair genes may predict neoadjuvant therapy response in triple negative breast patients. Genes Chromosom Cancer 55:915-924. https://doi.org/10.1002/gcc.22389

23. Werutsky G, Untch M, Hanusch C et al (2020) Locoregional recurrence risk after neoadjuvant chemotherapy: a pooled analysis of nine prospective neoadjuvant breast cancer trials. Eur J Cancer 130:92-101. https://doi.org/10.1016/j.ejca.2020.02.015

24. Jordan B, Jahn F, Sauer S, Jordan K (2019) Prevention and management of chemotherapy-induced polyneuropathy. Breast Care 14:79-84. https://doi.org/10.1159/000499599

25. Chen X, He C, Han D et al (2017) The predictive value of Ki-67 before neoadjuvant chemotherapy for breast cancer: a systematic review and meta-analysis. Futur Oncol 13:843-857. https://doi. org/10.2217/fon-2016-0420

26. Rouzier R, Perou CM, Symmans WF et al (2005) Breast cancer molecular subtypes respond differently to preoperative chemotherapy. Clin Cancer Res 11:5678-5685. https://doi. org/10.1158/1078-0432.CCR-04-2421

27. Haque W, Verma V, Hatch S et al (2018) Response rates and pathologic complete response by breast cancer molecular subtype following neoadjuvant chemotherapy. Breast Cancer Res Treat 170:559-567. https://doi.org/10.1007/s10549-018-4801-3

28. Harbeck N, Gluz O (2017) Neoadjuvant therapy for triple negative and HER2-positive early breast cancer. Breast 34:S99-S103. https ://doi.org/10.1016/j.breast.2017.06.038

29. Loibl S, Gianni L (2017) HER2-positive breast cancer. Lancet 389:2415-2429. https://doi.org/10.1016/S0140-6736(16)32417-5

30. von Minckwitz G, Procter M, de Azambuja E et al (2017) Adjuvant pertuzumab and trastuzumab in early HER2-positive breast cancer. N Engl J Med 377:122-131. https://doi.org/10.1056/nejmo a1703643

Publisher's Note Springer Nature remains neutral with regard to jurisdictional claims in published maps and institutional affiliations. 